Takashi Shibutani, ${ }^{1}$ Shiro Suzuki, ${ }^{2}$ Kohshiro Nakao, ${ }^{3}$ Takahiro Koyanagi, ${ }^{4}$ Shuko Tamaki, ${ }^{5}$ Lida Teng, ${ }^{6}$ Kazunori Honda, ${ }^{2}$ Yusuke Kajimoto, ${ }^{6,{ }^{*}}$ Ataru Igarashi ${ }^{6}$

'Hyogo Cancer Center, Akashi, Japan

${ }^{2}$ Aichi Cancer Center Hospital, Nagoya, Japan

${ }^{3}$ Gunma University Graduate School of Medicine, Gunma, Japan

${ }^{4}$ Jichi Medical University, Shimotsuke, Japan

${ }^{5}$ Saitama Medical University International Medical Center, Hidaka, Japan

${ }^{6}$ The University of Tokyo, Tokyo, Japan (ykajimoto234@gmail.com)

Objective: Costs related to cancer care negatively affect patients physically and psychologically. This is called financial toxicity (FT). To quantify FT, the "COmprehensive Score for financial Toxicity (COST)" tool has been developed in the United States. The COST tool consists of 11 items as patient-reported outcomes. Our objective is to validate the COST tool for patients with ovarian, cervical, and endometrial cancer. Additionally, we aim to assess reproducibility, which explains consistent scores over time by test-retest reliability.

Methods: In this multi-center study, patients were eligible for enrollment if they had ovarian, cervical or endometrial cancer; were receiving anti-cancer drug treatment for at least 2 months, and had out-of-pocket expenses for treatment at a hospital. Patients answered the COST tool twice and sent the responses to the data center by mail. Internal validity was examined by Cronbach's alpha at the first responses. Test-retest reliability was assessed by intra-class correlation coefficient (ICC).

Results: From April 2019 through July 2021, a total of 112 patients (ovarian cancer: 50, cervical cancer: 26 , and endometrial cancer: 36 ) answered the initial COST questionnaire and 99 patients answered the re-test. Median age was 58 years (range: $28-78$ years). Mean COST score was 19.82 $(\mathrm{SD}=7.28)$. Cronbach's alpha was 0.83 (95\% confidence interval $[\mathrm{CI}]=0.78-0.87)$. ICC was 0.85 (95\% CI=0.77-0.90).

Conclusion: The COST tool demonstrated good internal consistency and reproducibility among patients with gynecologic cancers. The results strengthen the evidence quality of FT researches using the COST tool in gynecologic cancers.

Poster (M08)

Gynecologic Cancer Screening

https://doi.org/10.3802/jgo.2021.32.S1.M08

\section{Association of cervicitis resulting from lower genital tract infections with oncogenic human papillomavirus and Pap smear abnormalities among Indian women}

Kavita V Anand," Sharmila A Pimple, Gauravi A Mishra

Department of Preventive Oncology, Centre for Cancer Epidemiology (CCE),
Tata Memorial Centre, Homi Bhabha National Institute, Mumbai, India (drkavitaanand@gmail.com)

Objective: To investigate the relationship between cervicitis with oncogenic Human papillomavirus, Bacterial vaginosis and epithelial cell abnormalities.

Methods: Total 508 women; 254 women with established diagnosis of cervicitis (case arm) and 254 asymptomatic women with healthy cervix (control arm) attending the tertiary cancer hospital for cervical cancer screening were enrolled in the study. Gram-stained smears for diagnosing lower genital reproductive tract infections (RTIs), Hybrid Capture-2 (HC2) test to diagnose oncogenic human papillomavirus (HPV) infection and Pap smears to demonstrate epithelial cell abnormalities (EP) were performed for all women enrolled in the study. Results: Study findings demonstrated the prevalence of bacterial vaginosis (BV), $\mathrm{HPV}$ infection and $\mathrm{EP}$ to be significantly higher among women in case arm (BV=44.09\%; $\mathrm{EP}=18.47 \%$; and $\mathrm{HPV}=14.17 \%)$ than control arm (BV=3.95\%; $\mathrm{EP}=4.82 \%$; and $\mathrm{HPV}=5.12 \%$ ). Case arm demonstrated increased risk for BV (odds ratio $[\mathrm{OR}]=19 ; 95 \%$ confidence interval $[\mathrm{CI}]=9.63-37.49)$, HPV $(\mathrm{OR}=3.06$; $95 \% \mathrm{CI}=1.58-5.92)$, and $\mathrm{EP}(\mathrm{OR}=4.47$; 95\% $\mathrm{CI}=2.30-8.68)$. Conclusion: Current study addresses the gaps in literature about the prevalence of HPV infection among Indian women with cervicitis. Our results demonstrate cervical inflammation to be significantly associated with increased risk of harboring HPV and EP. This results enforce the need of strengthening the RTI programs at national level in an attempt to reduce the burden of cervical cancer. Emerging trends of association of BV with cervicitis also warrant the treatment of $\mathrm{BV}$ in addition to treatment of recognized pathogens for cervicitis i.e., Gonococcal and Chlamydia infections as a part of syndromic management for women with cervicitis.

Poster (M09)

Miscellaneous

https://doi.org/10.3802/jgo.2021.32.S1.M09

\section{Comparison between International Ovarian Tumor Analysis (IOTA) - simple rules and risk malignancy indices in differentiating benign and malignant adnexal masses-prospective comparative study}

\author{
Vrushti Solanki, Pratibha Singh," Shashank Shekhar, \\ Navdeep Kaur Ghuman, Garima Yadav, Priyanka Kathuria, \\ Meenakshi Gothwal, Charu Sharma, Binit Sureka \\ All India Institute of Medical Sciences, New Delhi, India \\ (drpratibha69@hotmail.com)
}

Objective: To compare the sensitivity, specificity, and the 